\title{
Claves de interpretación de la realidad hispanoamericana (1)
}

Miguel Molina Martínez

Hablar de Hispanoamérica siempre es difícil, más cuando se trata de la Hispanoamérica actual. Es harto frecuente leer o escuchar interpretaciones acerca de su realidad enormemente apasionada. En verdad, en esas manifestaciones privan más las cuestiones de orden particular o subjetivo que un au téntico análisis de la situación.

Contribuyen a ello las especiales circunstancias que concurren en aquella región y que, de una forma y otra, incitan a la adopción de muy diversas posturas. Desde la constante inestabilidad política de gran parte de esos países - con todo lo que ello supone en el orden institucional, económico y social - hasta la presencia de fuertes intereses extranjeros, sin olvidar los frecuentes conflictos que enfrentan a las naciones hermanas, todo argumenta a favor de una aproximación a su ser repleta de escollos y dificultades. Resulta obvio que los planteamientos de acercamiento son múltiples como múltiple es la problemática que se aborda. Una problemática, además, cuyas repercusiones rebasan el propio marco hispanoamericano y alcanzan a todo el mundo.

A esas dificultades intrínsecas habría que añadir también un generalizado desinterés por no aclarar y resolver los problemas así como una cierta insinceridad por parte de quienes tienen ese cometido. Consecuencia de todo ello resulta ser esa difundida imagen de una Hispanoamérica pesimista donde poco se mejora y mucho se agrava. Hoy la preocupación por Hispanoamérica viene dada, en cierto modo, por su vitalidad o, en palabras de Julián Marías, por su potencialidad demográfica, por sus fermentos y por la capacidad de cambio que reviste su forma inestable.

No es mi objetivo en esta conferencia abordar en su totalidad la compleja realidad de Hispanoamérica. Su sólo intento desbordaría ampliamente las limitaciones de espacio y tiempo en que nos movemos. Al contrario, aquí me

(1) Conferencia pronunciada por el doctor Miguel Molina Martínez, Profesor Titular de Historia de América de la Universidad de Granada, en la Cámara de Comercio de Melilla el día 11 de octubre de 1984, con motivo de la Fiesta de la Hispanidad. 
propongo espigar únicamente algunos aspectos de dicha realidad que personalmente considero claves y sin que ello menoscabe la importancia y significado de los que se omiten.

Trataré, en primer lugar, del fenómeno demográfico y su espectacular crecimiento con todas sus consecuencias. En relación con ello, me detendré después en dos grupos de especial relevancia en el conjunto social: Uno, el indigena, de larga trayectoria y siempre presente en el devenir histórico hispanoamericano; el otro, el proletariado urbano, de más reciente formación pero, asimismo, de un significado incuestionable. Luego me ocuparé de uno de los fenómenos que mejor caracterizan aquella realidad: el militarismo o la constante intervención de las Fuerzas Armadas sobre los los poderes civiles. Un fenómeno que, lejos de erradicarse, presenta rasgos que confirman una vigencia indefinida. Por último, me referiré al papel de la Iglesia y al difícil compromiso adquirido. Punto éste de máxima actualidad en estos días al coincidir con el viaje de Juan Pablo II a tierras de Santo Domingo y Puerto Rico.

\section{La explosión demográfica}

El efecto más destacado en el desarrollo de la población de los países hispanoamericanos es la denominada explosión demográfica. Es el resultado de un aumento progresivo y constante de la tasa de natalidad y, a su vez, de una disminución de la tasa de mortalidad. Frente a una tasa de crecimiento alrededor del 1 ó 1,5 por 100 en los países desarrollados, Hispanoamérica sobrepasa con creces dichas cifras situándose en torno al 3 por 100 . Ello significa que algunas naciones duplicarán su población en el plazo de 20 años; plazo aún menor en el caso de Costa Rica si mantiene su 3,9 por 100 de crecimiento anual. Significa también que México, cuya capital supera ya los 12 millones de habitantes, incrementará su población en dos millones al año.

La magnitud del crecimiento es tal que Hispanoamérica pasó de $212 \mathrm{mi}-$ llones de habitantes en 1960 a 378 millones en 1980, estimándose que en el año 2000 se aproxime a la escalofriante cifra de 640 millones. El fenómeno es de tal envergadura y lleva aparejados tantos intereses que la no muy lejana Reunión de Cancún (México), convocada precisamente para discutir los problemas derivados de tal desarrollo demográfico, fue clausurada sin haber encontrado soluciones precisas al tema.

Como ya se ha apuntado, el aumento de la población es debido a dos causas principales: el incremento de los nacimientos y la disminución de la mortalidad. La primera presenta porcentajes muy altos, propios de países tercermundistas, en torno al 40-50 por mil. Conviene, no obstante, hacer la excepción de algunas regiones - Argentina, Uruguay, Chile-cuyas tasas de natalidad se aproximan más a los países europeos. La investigación en este 
campo viene a demostrar algunos hechos característicos. En primer lugar, una correlación entre nivel cultural y número de hijos. A menos grado de instruccion, mayor descendencia. En segundo lugar, la evidencia de que los indices de fecundidad disminuyen en las clases sociales altas $y$, en consecuencia, son las familias con menores ingresos las de mayor descendencia. Por último, existe una tasa de fecundidad más elevada en el campo que en la ciudad, efecto directo de la incidencia de la progresiva participación de la mujer en los puestos de trabajo.

En cuanto a la tasa de mortalidad, es realmente significativo su descenso generalizado a lo largo del siglo. Ello está relacionado con el mejor control y evitación de las epidemias, el retroceso de la mortalidad infantil y de las enfermedades endémicas tales como la malaria, el paludismo o las afecciones intestinales. Huelga decir que los avances de la medicina están en el fondo de esta cuestión. La batalla ganada a la mortalidad ha provocado profundos cambios en el proceso demográfico como son esa multiplicación veloz del número de habitantes o el incremento de la fertilidad, ya señalados, el rejuvenecimiento de la población y la mayor esperanza de vida. Aspectos que en ciertos paises tienen enorme repercusión amenazando con problemas de superpoblación y de pauperización. Por lo mismo, los respectivos gobiernos han de hacer frente a graves dificultades como las derivadas de la distribución del ingreso o de los costos del desarrollo: educación y salud pública, etc.

El rejuvenecimiento de la población, efecto inmediato de la disminución de la mortandad infantil, ha modificado la estructura demográfica que ahora representa de forma gráfica una pirámide de base muy ancha que se dispara hacia una cúspide pequeña. En otras palabras, en un breve período de tiempo se ha producido un suplemento de bocas a alimentar y necesidades realmente graves en el campo educacional que todavía hoy quedan muy lejos de encontrar respuestas adecuadas. Junto a ello, el no menos inquietante peligro de agitación social que supone la creciente mano de obra desocupada. El desfase entre crecimiento económico y crecimiento demográfico es en la actualidad una de las cuestiones más críticas que han de afrontar.

Es más, parece cernirse sobre este tema un principio de incompatibilidad según el cual los beneficios económicos no se podrán distribuir mientras no se detenga esa masiva incorporación de hombres y mujeres al mercado consumidor. El hecho es de una importancia vital ya que parece desprenderse de él la idea de que en Hispanoamérica no hay esperanzas de mejoramiento hasta que no se encauce la procreación.

Pero ahí radica otra cuestión espinosa. ¿Cómo hacerlo? Todos coinciden en que se debe disminuir la elevada tasa de natalidad sin que en la práctica se consiga nada positivo. Si en el siglo XIX toda Hispanoamérica tenía una política poblacional global, hoy no es asi y cada gobierno desarrolla una línea de actuación particular, en gran medida, determinada por la diversidad de condiciones.

El control de la natalidad está lejos de ser una solución óptima. Algunos 
gobiernos se muestran bastante reticentes para mostrar su apoyo. Las élites políticas y culturales se encuentran condicionadas por motivos religiosos que las predisponen en contra de su aceptación. Por su parte, los sectores rurales $e$ indígenas son igualmente reacios a estos postulados en la medida que pueden llegar a conocer o practicar tal medida. La enciclica Humanae Vitae ha jugado un papel decisivo a la que hay que anadir la actual postura del Vaticano muy precisa y contundente en el tema del control de la natalidad. Sólo el medio urbano y parte de la clase media parecen más permeables a la idea de la adopción de dichas medidas. Sin embargo, por su parcial implantación los efectos no son los que cabría esperar, máxime si se tiene en cuenta la cantidad de población que se incorpora anualmente al ciclo reproductor.

\section{Los sectores populares}

Sin pretender hacer un análisis de la estructura social hispanoamericana me referiré ahora a dos grupos de población cuya incidencia directa en el desenvolvimiento contidiano de la zona no ofrece discusión. Los conflictos y tensiones sociales que alli se suceden son de una magnitud tal que justifican el que les dedique algunas palabras.

$\mathrm{El}$ indígena es la clase secularmente explotada. Aunque bajo la presencia española gozó de un status jurídico reconocido y desarrollado en las Leyes de Indias, no cabe duda de que fue obligado a prestaciones personales de trabajo cuyas formas y modalidades variaron según épocas y regiones. Su situación a lo largo de esos tres siglos representó, en definitiva, el compromiso adquirido entre el paternalismo de los misioneros, la voluntad de control de los gobernantes y la presión de los colonos. Fue la Independencia la que rompió dicho estatuto y, lejos de avanzar hacia la incorporación total del indio a la vida política, supuso un notable retroceso del que todavia hoy no se ha recuperado.

En efecto, ni la guerra de independencia ni el período de caudillaje militar que siguió pudieron desarrollar -ni siquiera esbozar- una política progresista sobre el indio o la propiedad agraria. Al contrario, se inauguró un régimen que empeoraba la condición indígena. La nueva política abolía formalmente la mita, encomienda, etc., y comprendía medidas tendentes a la emancipación del indio como siervo. Pero, por otro lado, mantenía intactos el poder y la fuerza de los terratenientes al tiempo que dejaba desprotegidos a la pequeña propiedad y al trabajador de la tierra. De hecho, la abolición de la servidumbre indigena no pasaba de ser una mera declaración teórica.

Las reformas liberales de mediados del XIX no fueron sino otros tantos hitos hacia la ruptura de las tradiciones indias. Las desamortizaciones practicadas favorecieron en todas partes el latifundio a costa de la expropiación de las tierras tanto privadas como comunales que pertenecían al indio. Las 
posteriores reformas agrarias en poco o en nada han beneficiado a este sector de población. Aunque la Revolución Mexicana restableciera el ejido en un intento de recuperar la antigua estructura comunal agraria, la situación del indio es todavía hoy un problema a resolver. Tampoco Bolivia, Perú o Guatemala, por citar algunos casos, han logrado encarar la cuestión indígena de forma satisfactoria.

Las condiciones del indio, ocupado fundamentalmente en la agricultura, son duras y miserables. La explosión demográfica también se ha dejado sentir en su ámbito provocando una falta de tierra para laborar y forzando la salida fuera de su entorno tradicional. El primer efecto que se desprende de ello es la ruptura de su armazón institucional. El segundo, la falta de integración de estas masas emigrantes en el medio urbano donde se hacinan en los barrios margianles de la gran ciudad. En la actualidad, la crisis se agudiza. La comunidad indígena es incapaz de absorber una población creciente, máxime cuando su acceso a la propiedad de la tierra tropieza con innumerables obstáculos. La tradición cada vez se encuentra más amenazada por esta apertura al medio urbano mientras que la inevitable corriente moderna se deja ya sentir lamentablemente en regiones donde hasta hace pocas décadas se conservaba celosamente una cultura milenaria.

Políticamente el indio es un auténtico olvidado. Con frecuencia utilizado demagógicamente por unos y otros en campañas electorales, etc., apenas recibe otra atención de su gobierno. Ante tal situación, el indio suele responder de forma periódica con explosiones incontroladas de violencia que patentizan la situación dramática y límite que soportan. Invariablemente estas protestas son sofocadas también de forma violenta, seguidas de una dura represión en la que el indio se ve obligado a adoptar una actitud pasiva y servil hasta el nuevo brote revolucionario que inicia otro ciclo.

No existen grupos de presión netamente rurales pero sí algunas fuerzas latentes que podría ser más poderosas de estar mejor organizadas y orientadas. Pese a esta desorganización, en la actualidad el campesinado indígena se muestra cada vez más intransigente y reivindicativo. Sus protestas no responden, por lo general, a ningún plan preconcebido, ni están organizadas a nivel nacional. Es más, las guerrillas que surgen en muchos países con una ideología revolucionaria e inspiradas por elementos que provienen de las clases medias, no logran acaparar la atención de la totalidad del campesinado. Este, más que participar en ellas, las soporta.

En cuanto al proletariado industrial, hay que partir de la crisis de 1929 para que adquiera un volumen numéricamente significativo. Hasta entonces no se puede hablar de un auténtico proletariado industrial en Hispanoamérica; salvo las concentraciones mineras de México y Chile, la clase obrera permanece fraccionada y reducida al ámbito artesanal o pequeño taller aunque propicia, eso sí, a la recepción del anarquismo. Es la crisis de los años 30 y la consecuente industrialización por sustitución de importaciones la que impulsará la formación de un nuevo proletariado con rasgos bien definidos. 
De entre ellos hay que destacar, en primer lugar, el estar constituido por una población rural que desde el interior afluye a la ciudad y no ya por el emigrante europeo de etapas anteriores. Se trata, ante todo, de población sin conciencia de clase, ni experiencia sindical cuyo único objetivo es incorporarse a la vida urbana. A diferencia del proletariado europeo, educado en la ideología revolucionaria, éste repudia las organizaciones obreras existentes y busca la protección del Estado.

El fenómeno adquiere especial intensidad en los años 40 y 50 y explica el sindicalismo de masas de esa época con ejemplos tan señeros como el México de Lázaro Cárdenas, el Brasil de Getulio Vargas o la Argentina de Perón. Se trata de un sindicalismo burocratizado y controlado por un Estado que, en contrapartida, crea una legislación social avanzada y hace gala de una demagogia sin precedentes. No obstante, la propia dinámica del sistema, basado en un desarrollo económico boyante, será la causa de su propia destrucción. El éxodo rural a la ciudad aumenta atraido por las buenas expectativas que of rece pero llega un momento en que la industria es incapaz de absorber a toda la masa emigrante. La escasez de puestos de trabajo y el hecho de que el Estado encuentre serias dificultades para satisfacer todas las demandas provoca la radicalización del proletariado $y$, sobre todo, de aquella población no incorporada a la producción.

Puesto que el éxodo rural no se detiene y las industrias están cada día más imposibilitadas de ampliar su oferta de trabajo, los grupos marginales urbanos van alcanzando proporciones alarmantes y conflictivas. Dado su vertiginoso crecimiento y la condición de franca miseria en que vive, puede ser un buen fermento revolucionario, muy suceptible a la tentación de la guerrilla urbana y, en función de su origen rural, altamente sensible a la radicalización campesina. Un proletariado tan recientemente formado y procedente de sociedades arcaicas rurales es reacio a la implantación de un sindicalismo independiente decidado en exclusiva a la defensa de los intereses obreros. Pese a su falta de preparación representa hoy una fuerza política activa digna de tenerse encuenta. La concentración en determinadas regiones industriales próximas a la capital y su incidencia en el desarrollo económico le confieren un protagonisno nada desdeñable.

\section{El in tervencionismo militar}

Sin duda alguna, es el rasgo más peculiar y más difundido de la sociedad Hispanoamericana. No 'se trata de un fenómeno externo o accidental sino más bien una constante que convierte aquella región en la zona por excelencia de los golpes militares, de las juntas de oficiales o de las dictaduras de coroneles o de generales. Su estudio es tarea sumamente difícil por la variabilidad de sus manifestaciones, la complejidad de sus motivaciones o la diversidad de sus efectos y consecuencias. Quizá por todo ello sea uno de los temas 
que más bibliografía ha generado en los últimos tiempos. Un fenómeno de complicado encasillamiento que Ignacio Sotelo ha definido como el "predominio de los militares sobre las instituciones civiles que prevee la Constitución, debido al empleo o a la amenaza de empleo de la fuerza, para ocupar directamente el poder o influir en él".

Es una triste realidad que el desarrollo político de Hispanoamérica haga referencia siempre o esté marcado por el intervencionismo militar. Los militares no han dejado nunca de mezclarse en la vida política o de utilizar sus ejércitos para llegar al poder. Una ojeada retrospectiva sólo durante este siglo arrojaría el siguiente balance: Entre 1907 y 1957, en Venezuela únicamente hubo tres años de gobierno civil y en la República Dominicana seis. En América Central, salvo Costa Rica, hubo más gobiernos militares que civiles. Otro dato, en vísperas de la II Guerra Mundial todos los países, excepto cuatro, tenían gobiernos militares. Entre 1940 y 1960, se han sucedido una treintena de golpes militares. La década de los sesenta resulta especialmente significativa por la serie ininterrumpida de intervenciones golpistas: 1962, en Argentina y Perú; 1963, en Guatemala, Ecuador, República Dominicana y Honduras; 1964, en Brasil y Bolivia; 1966, en Argentina; 1968, en Perú y Panamá; 1969, en Brasil y Bolivia. Los años 70, de igual modo, han sido pródigos en este tipo de intervenciones. Ante tales hechos, para algunos sectores de opinión es un acto de fe la afirmación de que los pueblos hispanoamericanos son por naturaleza incapaces de gobernarse. No participo de esta impresión tan negativa a pesar de que su constante protagonismo o la incertidumbre de un golpe inmediato que viven hoy algunos paises haga pensar que se trata de un problema arraigado y sin visos de solución.

Sería presuntuoso por mi parte dar aquí una explicación de este fenómeno que en esencia es -ya se ha dicho-múltiple y vario. Por lo mismo, escapa a un encuadramiento formal rígido. Más útil considero esbozar una breve perspectiva histórica de su desarrollo con el ánimo de que se haga más comprensible su naturaleza o se repare en su diversidad.

Como punto de partida hay que desechar la tesis de que el militarismo es una herencia del pasado colonial español tal como han considerado algunos investigadores. Las reformas de la milicia emprendidas por Carlos III no explican en absoluto la atosigante presencia militar en los gobiernos hispanoamericanos en el XIX y XX. En segundo lugar, es preciso distinguir entre el caudillismo decimonónico y la intervención militar posterior. El caudillismo es la consecuencia directa de la falta de funcionamiento de las instituciones políticas tras la Independencia en 1825, favoreciendo por una serie de condiciones entre las que conviene destacar las siguientes: el fraccionamiento político sobre la unidad anterior; el desplazamiento del poder desde la ciudad al campo con la consiguiente ruralización de la vida y potenciación de la hacienda y del cacique; y la vida ficticia de la legalidad republicana. Todo ello crea el ambiente propicio para la intervención del caudillo quien mediante intrigas, promesas o concesiones obtiene el apoyo de una "clientela" que le 
sigue en la toma del poder. Este caudillo no tiene por qué ser militar aunque cuando triunfa se titula inmediatamente general. $\mathrm{El}$ ejército que le secunda no es un cuerpo orgánico ni institucionalizado. Se trata más bien de bandas armadas vinculadas por su carisma personal al jefe. Es obvio que en estas circunstancias no se puede hablar todavía de intervenciones militares tal como las entendemos en la actualidad.

El militarismo moderno sólo aparece a partir de finales del XIX y principios del $\mathrm{XX} \mathrm{y}$, en cualquier caso, acompañado de una profesionalización del ejército y de una modernización del equipo. Precisamente el desarrollo de la profesión militar organizada de forma regular, la formación de sus miembros en academias militares y el espíritu de cuerpo que les anima son las caracteristicas que diferencian a una y otra intervención.

En Chile es donde por primera vez se manifiesta el nuevo espíritu al iniciar la reorganización de su ejército con la ayuda de profesionales europeos. El general Koerner al frente de una misión militar alemana fue el artífice de la modernización del ejército chileno y de la mejora de la enseñanza militar según el modelo prusiano. El ejemplo fue seguido pronto por otros países. Bolivia y Argentina recibieron también a instructores alemanes mientras que Brasil, Ecuador, Perú y Paraguay se interesaron por los técnicos franceses. Por su parte, los EE.UU. se encargaron de la modernización de los ejércitos del Caribe. A principios del XX casi todas las naciones hispanoamericanas contaban con ejércitos profesionales fuertemente institucionalizados.

Esta transformación se opera en un marco determinado en lo social por la consolidación de la ligarquía terrateniente y en lo económico por una expansión basada en la exportación de materias primas y en una revolución en el sistema de transportes de la que no es ajena la creciente inversión extranjera. La misión del ejército en estos momentos es, por un lado, pacificar y unificar territorios para nuevas inversiones y, por otro, mantener la paz social interma necesaria para el desarrollo de los negocios. Integrado cada vez más por elementos de la clase media, el ejército se mantiene recluido en los cuarteles aunque apoyando a la oligarquía en el poder. Por un tiempo se pensó que el nuevo rumbo significaba el fin de la intervención golpista. Sin embargo, nada más lejos de la realidad. La profesionalización de la milicia no era sino la premisa para que el militarismo se mostrara con toda su fuerza.

En efecto, la crisis de 1929 fue el detonante que desencadenó toda una serie de intervenciones armadas. Al ponerse de manifiesto los defectos e inviabilidad del orden económico anterior y al cernirse sobre la oligarquía la amenaza de las clases medias y del proletariado, el ejército se convertirá en protagonista absoluto de la situación. Las Fuerzas Armadas intervienen, no obstante, para mantener el orden oligárquico en crisis y es la propia oligarquía la que solicita su ayuda para seguir en el poder. Este tipo de intervención, calificada por algunos de militarismo conservador, presenta características peculiares: a) el ejército se considera árbitro y garante del orden; b) actúa como institución; c) su acción queda justificada por un programa de 
regeneración nacional. Claro está que nación equivale a oligarquia; y d) su presencia en el poder se considera excepcional y contraria a las normas y, por lo mismo, es concebida por un tiempo limitado hasta que se restablezca el orden. Tal fórmula inevitablemente desencadenó una alternancia de gobiemos civiles y militares sin que ninguno de ellos lograr solucionar la crisis que requiere de cambios estructurales que nadie acomete.

Un intento de salvar dicha alternancia se da en Argentina con la introducción del llamado populismo militar. Su artífice, Juan Domingo Perón, se propone ir más allá del establecimiento del orden anterior y se justifica en función de una política de desarrollo nacional y de redistribución de la renta. A diferencia del modelo de gobierno militar precedente, cuenta con el apoyo entusiasta de las clases medias y, sobre todo, del proletariado urbano. La contradicción básica del sistema pronto quedará puesta de manifiesto. Su propósito de apoyar las reivindicaciones populares sin rozar los intereses de las clases dominantes sólo podía ser viable en épocas de expansión económica. Cuando se detiene tal expansión, cosa ya evidente en los años 50 , la fórmula peronista es incapaz de resistir y vuelve a retornar a la vieja alternancia gobierno militar-gobierno civil hasta 1966 en que los militares ocupan el poder para mantenerse en él ininterrumpidamente durante 17 años a los que pone fin la instauración de la democracia con Raúl Alfonsin.

Los años 60 contemplan la aparición de un nuevo militarismo de corte diferente a los anteriores y que bien puede calificarse de tecnócrata. Entre sus peculiaridades destacaría las siguientes: a) el ejército toma el poder como institución, huyendo del exacerbado personalismo innato al modelo populista; b) su misión no es restablecer el orden momentáneamente sino transformar las estructuras para el desarrollo económico y social. Por tanto, su presencia al frente de la nación se plantea de manera indefinida o a largo plazo; c) intransigencia con los partidos políticos a los que prohíbe o condena a una situación límite y d) el ejército discute en su interior y a puerta cerrada las distintas alternativas para presentarse luego al exterior como una fuerza unida e indiscutible. Como ejemplos representativos de esta nueva variante cabe citar el régimen implantado en Brasil en 1964, el peruano de Velasco Alvarado a partir de 1968 o las ya citadas juntas militares que se han sucedido en Argentina desde 1966 hasta 1983.

Al igual que en otras ocasiones esta modalidad de intervención refleja la incapacidad de las clases medias para afianzarse como grupo dominante sin la ayuda de los cuarteles. Esto es importante porque quizá radique aquí la clave del problema. Es decir, que sea la propia incapacidad de las diferentes clases para gobernar constitucionalmente la que provoca los golpes militares. Se suele hablar mucho de la excesiva participación del ejército en la vida política y ello hace olvidar un hecho de capital importancia: el que todas las clases sociales o los mismos partidos políticos se esfuerzan en provocar y alentar tales intervenciones cuando el gobierno de turno no es de su 
agrado. Puesto que dicha incapacidad tiene su origen en profundas contradicciones estructurales, que paradógicamente nadie entra a resolver, ta repetición de golpes militares en Hispanoamérica tiene visos de ser interminable.

\section{El papel de la Iglesia}

Sin ningún tipo de discusión la Iglesia en Hispanoamérica es una de las claves de interpretación más sobresalientes. Muchos de los acontecimientos que allf suceden necesitan de este componente para su correcta comprensión. Una profunda y larga tradición eclesiástica que se remonta a la misma época del Descubrimiento y que cuenta en su haber con una encomiable labor evangelizadora y cultural constituye el pilar más firme para que la Iglesia se haya convertido hoy en una fuerza de primerísimo orden.

Tres noticias entresacadas de la prensa de estos días pueden dar una idea aproximada del papel que desempeña en aquella región. Una de ellas, el viaje que en estos momentos realiza Juan Pablo II a Puerto Rico y Santo Domingo. La especial preocupación del Papa por la Iglesia hispanoaméricana revela sintomáticamente la vitalidad y la decisiva influencia que ejerce en todos los sectores de la población. Consciente de ello, el Vaticano está siempre atento a todo lo que en ella ocurre. La conmemoración del 12 de octubre que lleva a Juan Pablo II a los paises señalados inicia toda una serie de trabajos y nuevos viajes papales que confluyen en la fecha del V Centenario.

La segunda noticia habla de la oposición de la Iglesia chilena al régimen del general Pinochet. La negativa de algunos obispos de celebrar el anual Tedeum con el carácter político que hasta ahora se le ha venido dando es un claro signo de una nueva postura eclesiástica. El que tal acto se haya oficiado son la tradicional asistencia de las autoridades en un ambiente estrictamente religioso no deja de ser un importante revés para la dictadura que parece perder así a uno de sus grandes aliados. Si este hecho llega a ser noticia -en otros países no lo sería-, es precisamente por el determinante papel que la Iglesia desempeña en la vida política.

Por último, otro apunte más sobre su presencia viva. La Iglesia salvadoreña ha sido llamada como testigo e intermediaria en las conversaciones que mantendrá, José Napoléon Duarte, presidente de El Salvador, y la guerrilla para llegar a algún acuerdo sobre el fin de la contienda que divide al país.

Como se ve, más que en ningún otro punto del mundo la Iglesia hispanoamericana representa un factor de presión e influencia de enormes repercusiones. Es evidente que hoy los gobernantes consideran a los obispos como personajes oficiales cuya opinión debe ser tenida en cuenta. Con excesiva ligereza se la ha tachado de arcaica. Ciertamente durante la época colonial fue representante de un vigoroso conservadurismo social al servicio del gobierno. Sin embargo, tras la Independencia sus posiciones han variado considerable- 
mente hasta la actualidad donde le anima un espíritu abierto y se muestra a favor no ya de reformas sino de auténticas renovaciones estructurales. El compromiso militante de parte del clero o el rápido avance de la teología de la liberación son algunos aspectos de la nueva actitud eclesiástica.

El concilio Vaticano II fue decisivo para que se produjera tal cambio al potenciar la doctrina social de la Iglesia. Juan XXIII y Pablo VI fueron sus mejores portavoces. La encíclica Populorum Progressio y su condena del colonialismo hace pensar que fue redactada tomando como referencia la situación hispanoamericana. En cualquier caso, tuvo una resonancia especial en razón de la mayoría católica y subdesarrollada que allí vive. La defensa del proletariado rural y urbano, la lucha a favor de la justicia social e incluso la reforma agraria recibieron su espaldarazo en la II Conferencia de Medellín (1968) con la misma presencia de Pablo VI. El concilio espoleó a sectores importantes del clero hacia posturas progresistas e incluso revolucionarias. La actividad del arzobispo de Recife, Helder Cámara, en esta línea le dio prestigio internacional. Muchos sacerdotes son voceros de reivindicaciones sociales apareciendo enrolados en movimientos de tipo guerrillero como un Camilo Torres en Colombia.

La teología de la liberación es la expresión del nuevo comportamiento que parece poner fin a largas décadas de orientación conservadora. Hoy está siendo muy criticada y condenada por Juan Pablo II. Los teólogos de la liberación son interpelados en Roma sobre su ortodoxia en una especie de procesos inquisitoriales tan en desuso en estos tiempos. Los procesos al franciscano brasileño Leonardo Bolf y al sacerdote peruano, Gustavo Ramírez dejan bien a las claras los esfuerzos de la Santa Sede por frenar esta corriente.

La condena vaticana, basada en el hecho de cuestionarse las estructuras internas de la Iglesia a favor de un modelo más democrático y crítico, supone al mismo tiempo un rechazo al movimiento social que lleva implícito. Nelson Rockefeller, enviado por Nixon a Hispanoamérica en 1969 escribia en su informe final: "Debemos tener cuidado con la Iglesia latinoamericana pues si cumple los acuerdos de Medellín atenta contra nuestros intereses". Igualmente revelador es el Documento Santa $\mathrm{Fe}$ hecho público a principios de 1982 y básico en la política Reagan. En su proposición 3. a afirma: "La política exterior de E.E.U.U., debe comenzar a enfrentarse (y no a reaccionar con posterioridad) a la teología de la liberación. El papel de la Iglesia en América Latina es vital para el concepto de libertad política".

La reacción de la Santa Sede inevitablemente ha levantado tensiones en el seno de la comunidad eclesiástica americana y creado no pocas fisuras. El sacerdote ru ral sigue empeñado en la liberación de los fieles con los que convive sufriendo su misma penuria. Las altas jerarquías, por el contrario, y salvo algunas excepciones, están inclinadas a seguir las directrices vaticanas. En cualquier caso, la Iglesia hispanoamericana afronta una difícil situación. Ante todo, debe centrar bien su objetivo y evitar caer al servicio de fuerzas ajenas ya sean de un signo o de otro. En cuanto a la teología de la liberación 
yo le auguro posibilidades a pesar de las condenas papales. Existen circunstancias objetivas que la hacen necesaria y ante las que la Iglesia no puede permanecer insensible.

\section{Epilogo}

Larga es la lista de temas importantes que no he mencionado aquí -deficiente industrialización, guerrilla urbana y rural intervencionismo extranjero, etc. - Sin embargo, confío que las pocas cuestiones tratadas hayan evidenciado su carácter clave para la comprensión de la realidad hispanoamericana. Una realidad que necesita respuestas concluyentes pero que nunca llegan o cuando ocurre con insuficientes. Una realidad de difícil diagnóstico para la que no existen recetas seguras. Aquellos pueblos se debaten entre la miseria y el subdesarrollo, les agobia una creciente demografía, les atenaza un progresivo endeudamiento externo y viven bajo la amenaza del golpe militar y la dictadura: En fin, una situación explosiva de magnitud impredecible. Merece la pena reflexionar sobre estos hechos y que la dinámica, entre lo folklórico y lo serio, del V Centenario no nos distraiga de ello. 